\title{
New combination treatments in the management of diabetes: focus on sitagliptin - metformin
}

\author{
Jennifer Green \\ Mark Feinglos
}

Duke University Medical Center, Department of Medicine, Division of Endocrinology, Durham, North Carolina, USA
Correspondence: Jennifer B Green Duke University Medical Center, Division of Endocrinology, Metabolism, and Nutrition, DUMC Box 3222, Baker House - Room 280, Durham, NC 277I0, USA

$\mathrm{Tel}+\mid 9196845568$

$\mathrm{Fax}+19196817796$

Email green094@mc.duke.edu

\begin{abstract}
Type 2 diabetes mellitus is an increasingly prevalent condition worldwide. The complications of this disease are known to significantly increase the morbidity and mortality of those affected, resulting in substantial direct and indirect costs. Although good glycemic control has been shown to reduce the incidence and progression of diabetes-related microvascular complications, blood glucose levels are not adequately controlled in most individuals with diabetes. The reasons for this are many, and include issues such as poor adherence to complex medication regimes; costs of prescribed therapies; and the failure of traditionally prescribed medications to preserve beta cell function over time. However, our armamentarium of glucose-lowering drugs has expanded recently with the development of medications that act via the incretin pathway. Sitagliptin, the first commercially available dipeptidyl peptidase-4 inhibitor, inhibits the metabolism and inactivation of the incretin hormones GLP-1 and GIP. The subsequent elevation in levels of these hormones and associated prolongation of their actions has been shown to increase insulin secretion and suppress glucagon secretion in a glucoseappropriate fashion. Sitagliptin therapy in individuals with type 2 diabetes has been found to lower significantly hemoglobin A1c (Hblc) levels with a minimum of adverse side effects such as weight gain or hypoglycemia. Use of sitagliptin in conjunction with the insulin-sensitizing medication metformin has been shown to decrease HbA1c levels more significantly than does either drug alone. This combination of medications is generally well tolerated, with no adverse effects on weight and a very low likelihood of treatment-related hypoglycemia. Use of both drugs will positively affect many of the underlying metabolic abnormalities associated with type 2 diabetes, including the disordered secretion of insulin and glucagon as well as impaired sensitivity to insulin which are known to accompany this disease. Animal studies also suggest that dipeptidyl peptidase-4 inhibitor treatment may help to preserve beta cell mass; however, it is unclear at present whether or not this will prove to be the case in humans.
\end{abstract}

Keywords: diabetes, DPP-4, sitagliptin, metformin

\section{Introduction}

\section{Background and significance}

Diabetes mellitus is an increasingly prevalent condition worldwide. An estimated 20.8 million individuals in the US have diabetes, with the number of newly diagnosed adults nearly tripling between 1980 and 2005 (CDC 2008). The World Health Organization estimates that approximately 180 million individuals are affected worldwide, with this number expected to double by the year 2030 (WHO 2008). It is well understood that the financial and societal costs attributable to diabetes and its complications are substantial. In the US alone, estimated total costs associated with diabetes were estimated to be US\$132 billion in the year 2002 (CDC 2008). Diabetes-related microvascular complications are responsible for the majority of new cases of blindness, kidney failure, and nontraumatic amputations among US adults. Rates of stroke and heart-disease related deaths are 2-4 times higher in adults with diabetes than in the general population (CDC 2008). 
On a more positive note, intensive glycemic control will clearly slow the development and progression of many of these sequelae. This was convincingly demonstrated in the UK Prospective Diabetes Study (UKPDS), in which every $1 \%$ drop in hemoglobin A1c ( $\mathrm{HbA} 1 \mathrm{c})$ resulted in a $37 \%$ reduction in microvascular complications and a $21 \%$ reduction in any diabetes-related endpoint (Stratton et al 2000). As there was no threshold at which risk was not further reduced, it was hypothesized that a goal of normoglycemia might provide the best protection from these complications. As a result, increasingly strict recommendations for glycemic control have been issued by organizations such as the International Diabetes Federation (IDF) and the American Diabetes Association (ADA) (IDF 2008, ADA 2008). Despite this, most individuals with diabetes do not achieve optimal glycemic control. The National Health and Nutrition Examination Survey (NHANES) 1999-2000 found that only $37 \%$ of surveyed adults with diabetes had HbAlc values at the recommended ADA goal of $<7 \%$. Furthermore, only $7.3 \%$ of those individuals had achieved desirable control of the combined risk factors of glycemia, cholesterol, and hypertension (Saydah et al 2004). Although a variety of new glucose-lowering agents had become available in the interim, these percentages were unimproved compared with a similar survey performed in 1988-1994 (Saydah et al 2004).

It is clear that there are numerous barriers to the achievement of adequate glycemic control. Although always a cornerstone of appropriate diabetes management, lifestyle modification is unlikely to achieve either adequate or lasting control of hyperglycemia (Nathan et al 2006). Patient adherence to complex treatment regimes may be poor, and in fact appears to be inversely related to the number of medication doses that must be taken daily (Barnett 2004). Health care providers may also have a limited understanding of the degree of glycemic lowering likely to be accomplished by the addition of any particular class of medication, and often delay intensification of therapy until long after glycemic control has deteriorated. Most significant, however, may be the inability of traditionally utilized glucose-lowering therapies to sustain adequate glycemic control over time. In the previously noted UKPDS trial, treatment with diet, sulfonylurea, or metformin monotherapy adequately controlled diabetes for just half of the participants at 3 years, and only $25 \%$ of the participants at 9 years (Turner et al 1999). In the more recent ADOPT trial (A Diabetes Outcome Progression Trial), initial therapy with rosiglitazone resulted in greater durability of glycemic control than did treatment with metformin or glyburide (Kahn et al 2006). However, the significant expense of rosiglitazone coupled with concerns regarding the drug's cardiovascular safety have tempered the enthusiasm for its widespread use (Nissen and Wolski 2007).

\section{Normal glucose homeostasis}

The pancreatic islets are composed primarily of the beta cells, which produce insulin, and glucagon-producing alpha cells. In general, insulin is the hormone responsible for glucose disposal, glucagon for glucose availability. Under normal conditions these hormones work in concert to maintain glucose levels within a fairly narrow normal range. For example, food intake will promote insulin release, which will in turn increase formation of glycogen stores, facilitate glucose uptake in muscle and adipose tissues, and suppress hepatic glucose output, in part via paracrine suppression of glucagon release. The reduced glucagon secretion will limit hepatic glucose output through suppression of glycogenolysis and gluconeogenesis. The opposite series of events will occur during the fasting state, in which the effects of glucagon predominate and insulin secretion is minimal (Aronoff et al 2004).

\section{Pathophysiology of type 2 diabetes}

In type 2 diabetes, multiple perturbations of these regulatory processes are known. The disease is characterized by both insulin resistance and at least a relative degree of insulin deficiency. Factors such as aging, genetic predisposition, obesity, and lack of physical activity are all thought to contribute to the development of insulin resistance in peripheral and hepatic tissues. Although the beta cells of the pancreas initially compensate by increasing insulin secretion, individuals destined to develop diabetes will not be able to sustain insulin production at a level adequate to maintain normoglycemia. Interestingly, though, not all individuals with insulin resistance will develop frank diabetes. Some authors, in fact, suggest that beta cell dysfunction - rather than insulin resistance - is the primary metabolic defect in type 2 diabetes (Gerich 2002). This hypothesis is supported by studies in first-degree relatives of individuals with diabetes in which deficiencies in beta cell function have been demonstrated prior to the onset of hyperglycemia (Gerich 2002; Kahn 2003). Diabetes is also associated with a loss of normal oscillatory insulin release in response to meals; in particular, studies have demonstrated that the first phase of insulin secretion (which serves to "prime" insulin-sensitive tissues to the influx of glucose associated with meals) is often 
absent in individuals with diabetes (Kahn 2003; Del Prato and Marchetti 2004). Inadequate suppression of glucagon secretion is known to contribute further to the resultant hyperglycemia (Aronoff et al 2004).

Although insulin resistance is a hallmark of type 2 diabetes, the contribution of beta cell dysfunction to hyperglycemia cannot be underestimated. Data from studies such as the UKPDS suggest that beta cell function is generally diminished by more than $50 \%$ by the time an individual is diagnosed with diabetes (UKPDS 1995). In the UKPDS trial, progressive beta cell dysfunction was also noted in the years subsequent to diagnosis despite intervention with lifestyle modification and/or treatment with oral glucose-lowering medication. This deterioration in beta cell function likely contributes significantly to the difficulty in maintaining adequate glycemic control over time.

\section{Role of the incretin hormones}

Our understanding of islet physiology has increased substantially with the discovery of the gut-derived incretin hormones. The so-named "incretin effect" has been demonstrated for years, and refers to the fact that an oral glucose load will stimulate insulin production to a greater degree than will glucose administered intravenously. Importantly, it is understood that the incretin effect is diminished in individuals with type 2 diabetes (Nauck et al 1986). The enhanced insulin secretion achieved through oral glucose loading is now understood to be due to the food-stimulated release of gut-derived hormones. The most important of these hormones are glucagon-like peptide-1 (GLP-1) and glucose-dependent insulinotropic peptide (GIP) (Aronoff et al 2004). GLP-1 (secreted by L cells of the small intestine) has a variety of effects on glucose homeostasis, including regulation of gastric emptying; the stimulation of insulin secretion in a glucose-dependent fashion; and the suppression of glucagon secretion (Drucker 2003). Both GLP-1 and GIP are rapidly degraded to largely inactive metabolites by the enzyme dipeptidyl peptidase-4 (DPP-4) (Drucker 2003). Individuals with type 2 diabetes are GLP-1 deficient; however, infusion of GLP-1 to persons with diabetes has been shown to lower both postprandial and fasting blood glucose levels (Drucker 2003; Aronoff et al 2004). Nauck et al (1993) have demonstrated that GLP-1 administration to individuals with type 2 diabetes will facilitate glucose lowering via glucosedependent stimulation of insulin secretion and suppression of glucagon levels. Secreted by K cells of the small intestine, GIP is also known to stimulate insulin secretion; individuals with type 2 diabetes appear to have a diminished response to this hormone, rather than being deficient in its production (Holst 2002; Drucker 2003).

\section{Challenges and strategies in diabetes management}

The optimal approach to glycemic management in type 2 diabetes remains unclear, as the most commonly prescribed diabetes therapies have proven largely ineffective in maintaining glucose control long term. A set of recently published guidelines are more aggressive than most, in that medical therapy with metformin is recommended (along with lifestyle modification) at the time of diabetes diagnosis (Nathan et al 2008). However, based upon the UKPDS experience, it seems unlikely that this strategy alone will alter the known progression of beta cell dysfunction in type 2 diabetes. Ideally, new therapies for diabetes management will effectively and adequately lower blood glucose, and increase the durability of that control once achieved. Our ever-expanding armamentarium of medications may make these goals more easily manageable. Effective glycemic control will likely require the use of medications that target both beta cell dysfunction and insulin resistance; furthermore, in order to maintain glycemic control over the long term, interventions that preserve beta cell function should probably be initiated early in the disease process. Although the most commonly prescribed diabetes medications are generally those in the insulin secretagogue and insulin-sensitizing categories, newer agents that utilize the incretin pathway offer exciting alternatives and may significantly alter future diabetes management.

A variety of antihyperglycemic agents with differing and complementary mechanisms of action are currently available. Thoughtful combinations of these medications allow the prescriber to target many of the physiologic abnormalities which are intrinsic to type 2 diabetes. Many studies to date suggest that combinations of different classes of oral antidiabetic drugs are more effective than are maximal doses of a single drug; in fact, many authors now suggest the use of combination therapy early - if not initially - in the disease process (DeFronzo 1999; Inzucchi 2002; Charpentier 2002; Riddle 2005). Combination therapy, in addition to being more effective than monotherapy, will often permit the utilization of submaximal doses of the agents used. Therefore, patients will derive the benefits of all drugs used, while minimizing the likelihood of intolerable side effects from any one class (Bell 2004). Several combination tablets are now available which help to defray the cost of diabetes management, and may improve adherence to 
prescribed therapy. In the remainder of this article, information regarding the mechanism of action, safety, and efficacy of the DPP-4 inhibitor sitagliptin will be reviewed, with a particular emphasis on its use in combination therapy with metformin. Studies thus far indicate that combinations of these two drugs are effective in blood glucose lowering, and may offer a more desirable side effect profile than that seen with other classes of antihyperglycemic agents.

\section{Mechanisms of glycemic control Sitagliptin}

Sitagliptin is one of several orally administered agents recently developed or in development which have been shown to inhibit the activity of the DPP-4 enzyme. This enzyme circulates in a soluble form in plasma, and is expressed in a wide variety of tissues including the kidney, liver, lung, and lymphocytes (Drucker and Nauck 2006). Importantly, this enzyme is responsible for the rapid degradation and inactivation of the incretin hormones GLP-1 and GIP. DPP-4 inhibition increases levels of and extends the half-life of these circulating incretins, thereby prolonging their actions (Ahren 2007). GLP-1 levels after DPP-4 inhibition are increased, resulting in stimulation of insulin secretion and suppression of glucagon secretion in a fashion which is glucose-dependent (ie, glucose-appropriate). DPP-4 mediated inhibition of glucagon secretion contributes to improved glucose homeostasis through suppression of hepatic glucose output. Inhibition of the enzyme has been shown to increase HOMA-B (homeostatic model assessment of beta cell function) and decrease the proinsulin/insulin ratio, suggestive of an increased insulin secretion which is accompanied by improvements in insulin production and processing (Raz et al 2006). Interestingly, rodent studies suggest that DPP4 inhibition may preserve beta cell mass; however, there are no data at present to support a similar effect in humans (Mu et al 2006). GIP levels are also increased via DPP-4 inhibition - the clinical significance of this is unclear, though, as individuals with type 2 diabetes appear to have decreased responsiveness to this incretin (Ahren 2007). The likelihood of hypoglycemia due to sitagliptin monotherapy is low, as the activity of the medication depends on hormones that exert their effects in a glucose-dependent manner.

\section{Metformin}

The precise mechanisms through which metformin exerts its glucose lowering effects are not entirely understood. However, its primary mode of action appears to be that of increasing hepatic insulin sensitivity, resulting in decreased hepatic glucose output through suppression of gluconeogenesis and glycogenolysis (Goodarzi and Bryer-Ash 2005). Metformin may also modestly augment glucose uptake in peripheral tissues, increase fatty acid oxygenation, and increase glucose metabolism in the splanchnic bed (Bailey 2005). Metformin's effects appear to be at least in part mediated by adenosine monophosphate-activated protein kinase (AMPK), but it is unclear if this pathway represents the drug's specific or unique target (Reitman and Schadt 2007). Administration of metformin to obese subjects was also found to increase levels of active GLP-1 after a glucose load: this phenomenon appears to occur through mechanisms other than DPP-4 inhibition, and may instead be due to direct stimulation of GLP-1 secretion or a reduction in DPP-4 secretion (Mannucci et al 2001; Lenhard et al 2004). Importantly, the likelihood of hypoglycemia induced by metformin monotherapy is quite low, as the drug does not exert its effects through an increase in insulin secretion.

\section{Pharmacology/pharmacokinetics Sitagliptin}

Sitagliptin is an orally bioavailable and active inhibitor of the DPP-4 enzyme, which may be administered with or without food. The drug is highly selective for DPP-4, with markedly greater affinity for that enzyme than for the related enzymes DPP-8 and DPP-9 (Herman et al 2005). Sitagliptin rapidly inhibits the activity of DPP-4 in a dose-dependent fashion, with doses of $50 \mathrm{mg}$ and $100 \mathrm{mg}$ inhibiting the enzyme by $80 \%$ over 12 and 24 hours, respectively. Inhibition of DPP-4 activity by $80 \%$ results in a 2 - to 3 -fold increase in active GLP-1 levels, and is the level of inhibition at which near maximal glucose lowering is seen. Of the administered dose, $79 \%$ is excreted unchanged in the urine, via active tubular secretion (Herman et al 2005, 2007). The potential for adverse interactions with other medications is low, as the drug is minimally metabolized and does not appear to inhibit or induce cytochrome P450 enzymes. In particular, no alterations in the pharmacokinetics of other oral hypoglycemic agents (including rosiglitazone, glyburide, and metformin) have occurred after sitagliptin administration (Ahren 2007; Herman et al 2007). The usual recommended dose of sitagliptin is $100 \mathrm{mg}$ daily; however, as renal insufficiency has been found to increase drug exposure, the dose should be reduced in individuals with modest or severe degrees of renal dysfunction. It is recommended that the daily dose be reduced to $50 \mathrm{mg}$ daily if the creatinine clearance is less than $50 \mathrm{~mL} / \mathrm{min}$, and reduced further to $25 \mathrm{mg}$ daily if the creatinine clearance is less than $30 \mathrm{~mL} / \mathrm{min}$. Patients who 
are prescribed sitagliptin therapy should have renal function assessed prior to and periodically during therapy, in order to ensure appropriate dosing (Merck and Co, Inc. 2007).

\section{Metformin}

Metformin is an orally administered medication which is 50\%-60\% bioavailable. Administration with food may decrease its absorption, the clinical significance of which is unknown. The drug is minimally protein-bound, and has few known drug interactions other than that known to occur with cimetidine, which increases metformin levels in plasma by up to $40 \%$. The drug has an elimination half-life (t1/2) of approximately 6 hours. It is generally dosed 2-3 times daily, but is available in an extended release preparation which may be administered once a day. Eighty-five percent of the maximal glucose-lowering effect is seen at a daily dose of $500 \mathrm{mg} 3$ times daily, with a total daily dose of $2000 \mathrm{mg}$ exerting the most effective glucose lowering (Scheen 1996; Bailey 2005).

Metformin is not metabolized prior to its complete excretion in the urine via glomerular filtration and tubular secretion. Decreases in renal function will decrease clearance of the medication.

Metformin accumulation has been associated with lactic acidosis: although this is a rare complication of therapy, the condition is fatal $50 \%$ of the time. The true likelihood of lactic acidosis occurring as a result of metformin accumulation is unclear. Although the incidence is reported to be 0.03 cases per 1000 patient-years of use, a recent systematic review of data from 206 trials of metformin therapy (47,846 patientyears of use between 1959-2005) found no cases of fatal or nonfatal lactic acidosis to have occurred in conjunction with the drug (Salpeter et al 2006). However, given these concerns, the drug is contraindicated in the setting of renal dysfunction (males with a creatinine over $1.5 \mathrm{mg} / \mathrm{dL}$, and females with a creatinine above $1.4 \mathrm{mg} / \mathrm{dL}$ ), or in those at risk for lactic acidosis due to hypoperfusion of the kidney or other tissues. This includes individuals with a history of congestive heart failure requiring medical treatment, significant hepatic dysfunction, or metabolic acidosis. The drug should also be discontinued pre-operatively, or in the setting of sepsis or an acute myocardial infarction. The drug must be discontinued prior to the administration of radiocontrast dye, and should be restarted only if renal function remains within an acceptable range at 48 hours after the procedure. Renal function must be assessed prior to and periodically during metformin therapy, particularly in the elderly in whom it is recommended that creatinine clearance be assessed in order to most accurately detect any significant degree of renal dysfunction (Scheen 1996; Bailey 2005).

\section{Medication efficacy and durability Metformin}

As a large number of studies have included the use of metformin for glucose lowering, a complete review of this literature is beyond the scope of this article. In general, though, it is expected that metformin monotherapy for type 2 diabetes will result in $\mathrm{HbA} 1 \mathrm{c}$ lowering of approximately $1.5 \%-2 \%$ (Goodarzi and Bryer-Ash 2005). Metformin monotherapy as studied in the UKPDS trial resulted in sustained glucose lowering for over 2 years on average; however, a secondary failure rate of $5 \%-10 \%$ per year was noted. This failure rate was comparable to that seen with sulfonylurea therapy, and likely reflects a progressive loss of beta cell function which was not ameliorated with the use of metformin (UKPDS 1998; Bailey 2005). Despite this deterioration in glycemic control over time, a reduction in major cardiovascular endpoints was seen in a group of obese UKPDS subjects treated preferentially with metformin (UKPDS 1998). As this was not clearly due to better glycemic control, it is theorized that this benefit was due to unique favorable effects of metformin upon the cardiovascular system. For example, metformin therapy does not cause weight gain, and may in fact result in weight loss with an associated reduction in blood pressure. Although it is generally felt to be lipid neutral, metformin administration has been found to reduce levels of triglycerides, total cholesterol, and LDL cholesterol in some clinical trials. Furthermore, metformin therapy may result in desirable physiologic changes including vasodilatation, increased fibrinolysis, and decreased platelet aggregation (Goodarzi and Bryer-Ash 2005). Metformin therapy has been proven useful in diabetes prevention, as its use in the Diabetes Prevention Program trial reduced the annual incidence of type 2 diabetes from $11 \%$ to $7.8 \%$ per year in patients with impaired glucose tolerance (Knowler et al 2002). It has recently been recommended that metformin therapy be initiated at the time of diagnosis of type 2 diabetes, in conjunction with lifestyle modification (Nathan et al 2008).

\section{Sitagliptin}

Seven large phase III trials of sitagliptin as monotherapy or as part of a combination of drugs to treat type 2 diabetes have been published. Two similar studies of sitagliptin monotherapy enrolled 741 and 521 patients into 24-week and 18-week treatment periods. These study populations had mean baseline $\mathrm{HbA} 1 \mathrm{c}$ levels of $8.0 \%$ and $8.1 \%$ respectively. 
In both trials, patients were randomized to treatment with sitagliptin $100 \mathrm{mg}$ daily, sitagliptin $200 \mathrm{mg}$ daily, or placebo. In the 18-week trial, sitagliptin $100 \mathrm{mg}$ therapy reduced $\mathrm{HbA} 1 \mathrm{c}$ by $0.6 \%$ compared with placebo, while the same dose reduced $\mathrm{HbA} 1 \mathrm{c}$ by $0.8 \%$ in the 24 -week study. No significant difference in efficacy was seen between the $100 \mathrm{mg}$ and $200 \mathrm{mg}$ dosing groups in either trial; thus only the $100 \mathrm{mg}$ dose has been used in subsequent studies. Low rates of hypoglycemia, stable body weight, and improvement in markers of beta cell function were seen with sitagliptin therapy in both monotherapy studies (Achsner et al 2006; Raz et al 2006).

Analysis of the efficacy of sitagliptin therapy in addition to the thiazolidinedione pioglitazone was performed in a group of 353 individuals with a mean baseline $\mathrm{HbA} 1 \mathrm{c}$ of $8.0 \%$. These individuals, on pre-existing therapy of pioglitazone 30 or $45 \mathrm{mg}$ per day, were randomized to receive add-on therapy with sitagliptin $100 \mathrm{mg}$ daily or placebo for a 24-week treatment period. The addition of sitagliptin resulted in a mean reduction in $\mathrm{HbA} 1 \mathrm{c}$ of $0.7 \%$ compared with placebo. Furthermore, significant reductions in mean fasting blood glucose levels and improvements in markers of beta cell function were seen with active therapy compared with placebo. Sitagliptin therapy did not significantly change in mean body weight compared with either baseline weight or the placebo group (Rosenstock et al 2006).

One 52-week noninferiority trial has been performed which compared the efficacy of sitagliptin versus glipizide as add-on therapy to metformin. This trial of 353 individuals with a mean baseline $\mathrm{HbA} 1 \mathrm{c}$ of $7.5 \%$ randomized patients to either sitagliptin $100 \mathrm{mg}$ daily or glipizide $5 \mathrm{mg}$ daily (uptitrated to a maximum of $20 \mathrm{mg}$ daily), in addition to ongoing metformin treatment at a total daily dose of at least $1500 \mathrm{mg}$. At 52 weeks, it was found that treatment with either sitagliptin or glipizide (at an average daily dose of $10 \mathrm{mg}$ ) resulted in a significant mean reduction in $\mathrm{HbA} 1 \mathrm{c}$ of $0.7 \%$, thus establishing noninferiority. Of note, sitagliptin therapy was associated with less hypoglycemia and a more favorable effect on weight than was glipizide. In particular, the sitagliptin group lost an average of $1.5 \mathrm{~kg}$, while the glipizide group sustained a mean weight gain of $1.1 \mathrm{~kg}$ (Nauck et al 2007).

\section{Sitagliptin and metformin combination therapy}

Charbonnel et al performed a 24-week study of sitagliptin and metformin combination therapy in 701 subjects with a mean $\mathrm{HbA} 1 \mathrm{c}$ of $8.0 \%$. In this trial, participants were randomized in a 2:1 ratio to sitagliptin $100 \mathrm{mg}$ daily or placebo, as adjunctive therapy to ongoing metformin treatment of at least $1500 \mathrm{mg}$ daily. Sitagliptin therapy significantly reduced $\mathrm{HbA1c}$ by $0.7 \%$ compared with placebo, an effect that was sustained throughout the treatment period. Active therapy also resulted in a significantly greater percentage of individuals achieving an $\mathrm{HbA} 1 \mathrm{c}$ goal of less than $7 \%$ compared with placebo $(47 \%$ vs $18 \%$ ); provided significant reductions in mean fasting and 2-hour glucose levels; and increased markers of beta cell function. Specifically, improvements in fasting insulin, c-peptide, proinsulin/insulin ratio, and HOMA-B were noted to be significant with sitagliptin therapy compared with placebo. Sitagliptin therapy did not increase risk of hypoglycemia or adverse gastrointestinal side effects compared with placebo, and no significant difference in mean body weight was noted (Charbonnel et al 2006).

A large study by Goldstein et al (2007) analyzed the effects on glycemic control of various doses of metformin, sitagliptin, combinations of both drugs, or placebo over 24 weeks in 1091 individuals with baseline HbA1c values of $7.5 \%-11 \%$ (mean $8.8 \%$ ). Participants were randomized to one of six treatment regimes: sitagliptin $100 \mathrm{mg} / \mathrm{metfor}-$ min $2000 \mathrm{mg}$ daily; sitagliptin $100 \mathrm{mg} / \mathrm{metformin} 1000 \mathrm{mg}$ daily; metformin $2000 \mathrm{mg}$; metformin $1000 \mathrm{mg}$ (all dosed in twice daily, evenly divided doses); sitagliptin $100 \mathrm{mg}$ daily; or placebo. All active therapies as outlined above resulted in mean $\mathrm{HbA} 1 \mathrm{c}$ reductions of $2.07 \%, 1.57 \%$, $1.30 \%, 0.99 \%$, and $0.83 \%$ respectively compared with placebo. Of note, an additional open-label analysis of individuals with baseline $\mathrm{HbA} 1 \mathrm{c}$ values greater than $11 \%$ demonstrated that treatment with sitagliptin $100 \mathrm{mg} / \mathrm{metformin}$ $2000 \mathrm{mg}$ daily produced a within-group $\mathrm{HbA} 1 \mathrm{c}$ reduction of $2.9 \%$. In all treatment groups, the improvements in fasting blood glucose and $\mathrm{HbA} 1 \mathrm{c}$ values remained relatively stable after reaching a nadir at 6 weeks. Administration of combined sitagliptin and metformin increased HOMA-B more significantly than did sitagliptin or metformin monotherapy. Rates of hypoglycemia with active therapy were low (ranging from $0.5 \%$ to $2.2 \%$ ), and not significantly different from placebo $(0.6 \%)$. Reductions in mean body weight were small but significant in all groups other than the sitagliptin monotherapy group, in which mean body weight remained stable at 24 weeks. Rates of adverse gastrointestinal side effects with combination therapy were similar to those reported with comparable dosing of metformin monotherapy (Goldstein et al 2007).

Finally, Hermansen et al performed a 24-week analysis of sitagliptin versus placebo therapy in patients inadequately 
controlled on glimepiride \pm metformin therapy. These 441 participants had a mean baseline $\mathrm{HbA} 1 \mathrm{c}$ of $8.34 \%$, and were randomized to sitagliptin $100 \mathrm{mg}$ daily or placebo in addition to baseline therapy with glimepiride or glimepiride plus metformin. At 24 weeks, sitagliptin treatment had significantly reduced $\mathrm{HbA} 1 \mathrm{c}$ by $0.74 \%$ overall compared with placebo, with the most pronounced mean reduction in HbA1c $(0.89 \%)$ seen in the group that had been treated with all three active drugs. In this trial, sitagliptin treatment resulted in a higher number of adverse events compared with placebo, due primarily to an increased rate of nonsevere hypoglycemia. Overall rates of hypoglycemia were $12.2 \%$ with sitagliptin, compared with $1.8 \%$ for placebo - most episodes could be explained by precipitating factors such as fasting or delayed meals. A mean increase in body weight of $0.8 \mathrm{~kg}$ was noted in the sitagliptin group, while the placebo group lost an average of $0.4 \mathrm{~kg}$. Sitagliptin therapy appeared to be lipid neutral compared with placebo in this trial (Hermansen et al 2007).

\section{Safety and tolerability}

Sitagliptin therapy appears to be safe and well-tolerated by most, as outlined in the previous sections. When administered as monotherapy or in combination with agents other than sulfonylureas, sitagliptin therapy appears unlikely to cause hypoglycemia and is generally weight-neutral. However, when administered concomitantly with a sulfonylurea, rates of hypoglycemia and weight gain are significantly greater than that seen with placebo. Other adverse effects noted to occur in clinical trials of DPP-4 inhibition have included increased reports of nasopharyngitis, upper respiratory infection, and headache - these were not likely to be severe or result in discontinuation of the medication. As previously noted, dosing of sitagliptin should be adjusted in persons with moderate or severe renal dysfunction; however, its use is not contraindicated in the setting of renal dysfunction. In the postmarketing period, reports of serious hypersensitivity reactions in conjunction with sitagliptin therapy have occurred - the causal relationship of the drug to these complications is unclear at present. These reactions have included cases of anaphylaxis, angioedema, and exfoliative skin conditions and thus the use of sitagliptin is contraindicated in individuals who have experienced a previous hypersensitivity reaction to the drug (Merck and Co, Inc. 2007).

Although sitagliptin may be used safely in the setting of renal dysfunction, the use of metformin in such a situation would be contraindicated. Thus it is imperative that the safety profile of each drug be reviewed prior to prescription of a sitagliptin/metformin combination. Renal function must be assessed prior to and periodically during the use of both of these medications in order to ensure appropriate dosing (sitagliptin) and safety of use (metformin). The most commonly reported adverse side effects associated with metformin therapy are gastrointestinal, and include abdominal pain, diarrhea, and anorexia - these side effects are estimated to occur in approximately $20 \%$ of metformin users (Goodarzi and Bryer-Ash 2005). Generally these side effects may be minimized by initiating a low dose of metformin, gradually uptitrating the metformin dose, and administering the medication with meals. Of note, the addition of sitagliptin to metformin therapy does not appear to cause a greater incidence of gastrointestinal side effects than does metformin alone. Other side effects associated with metformin therapy include reductions in vitamin B12 levels, and reports of a metallic taste in the mouth (Goodarzi and Bryer-Ash 2005). Both sitagliptin and metformin are pregnancy category B. At present, inadequate information is available for either drug with which to make recommendations for use in pregnancy, lactation, or pediatric populations.

\section{Quality of life, satisfaction, and acceptability}

Sitagliptin and metformin are medications that should be readily acceptable to most individuals with type 2 diabetes. Both are oral agent medications, which are generally preferred to injection therapies and require no special supplies for administration. Adherence and patient satisfaction may also increase with utilization of the newly available sitagliptin/metformin combination tablet. In addition to the increased convenience of a combination tablet, such medications often help to defray the costs of antihyperglycemic therapies. The gastrointestinal side effects associated with metformin therapy - although experienced by a significant percentage of metformin users - can generally be minimized through slow upward titration in dosing and administration of the medication with meals, and do not seem more likely to occur when sitagliptin is used in conjunction with metformin therapy. Additionally, these agents are unlikely to cause hypoglycemia when administered individually or in combination. Most attractive, perhaps, would be the effects of these two medications (or lack thereof) on body weight. Sitagliptin appears to be largely weight-neutral, and does not appear to interfere with any weight loss that might be conveyed by metformin therapy. The exception to this, however, appears to be when sitagliptin is added to sulfonylurea therapy: use 
of that particular combination has been associated with increases in hypoglycemia and weight gain.

\section{Conclusions and place in therapy}

Type 2 diabetes mellitus and the many complications thereof represent a major public health concern worldwide. Although the benefits of good glycemic control have been well documented, the majority of those affected by the disease have either not reached or maintained desirable levels of HbAlc. The reasons for this are multifactorial, and are at least partly explained by the inability of traditionally used drugs to arrest the progressive loss of beta cell function over time. Some of the factors contributing to poor glycemic control may perhaps be more easily remedied than others. Examples of potentially helpful strategies might include the use of medications that are dosed infrequently or in combination tablets to improve patient adherence; use of medications that are financially accessible to patients; and medications with low rates of unpleasant side effects which might lead to drug discontinuation. Whether or not any of the newer classes of antihyperglycemic medications will prove useful in the maintenance of beta cell mass remains to be seen; however, some preliminary data suggest that this may be the case with DPP-4 inhibitors such as sitagliptin.

The combination of sitagliptin and metformin in type 2 diabetes management has been shown in clinical trials to be effective in blood glucose lowering, with very low associated rates of hypoglycemia and no attenuation in the potential weight loss effects seen with metformin monotherapy. Use of this combination fits nicely with a recently published algorithm of recommended drug therapy for diabetes, as the strategy suggested by Nathan et al (2008) suggests initiation of metformin at the time of diagnosis and subsequent rapid intensification of drug therapy should inadequate glycemic control persist. It is furthermore not unreasonable to assume that the use of sitagliptin/metformin combination therapy as a first-line intervention would result in more effective blood glucose lowering than would metformin therapy alone. In addition, combination therapy implementing less than maximal doses of metformin might permit a significant degree of glucose lowering with a minimum of metformin-associated gastrointestinal effects. Of note, though, the introduction of a sulfonylurea to this drug combination appears to be associated with an increased likelihood of hypoglycemia as well as weight gain. Clearly, long-term studies will be needed to assess definitively the effectiveness of sitagliptin/metformin therapy in preserving beta cell mass and function over time, as well as any potential impact that this combination might have on the development of cardiovascular complications.

\section{Disclosures}

Neither author has any conflicts of interest to disclose.

\section{References}

Ahren B. 2007. Dipeptidyl peptidase-4 inhibitors. Diabetes Care, 30:1344-50.

[ADA] American Diabetes Assocation. 2008. Standards of medical care in diabetes - 2008. Diabetes Care, 31(S1):S12-S54.

Aronoff SL, Berkowitz K, Shreiner B, et al. 2004. Glucose metabolism and regulation: beyond insulin and glucagon. Diabetes Spectrum, 17:183-90.

Aschner P, Kipnes MS, Lunceford JK, et al. 2006. Effect of the dipeptidyl peptidase-4 inhibitor sitagliptin as monotherapy on glycemic control in patients with type 2 diabetes. Diabetes Care, 29:2632-7.

Bailey CJ. 2005. Treating insulin resistance in type 2 diabetes with metformin and thiazolidinediones. Diabetes Obes Metab, 7:675-91.

Barnett AH. 2004. Treating to goal: challenges of current management. Eur $J$ Endocrinol, 151(S2):T3-7; discussion T29-30.

Bell DS. 2004. Practical considerations and guidelines for dosing sulfonylureas as monotherapy or combination therapy. Clin Ther, 26:1714-27.

[CDC] Centers for Disease Control and Prevention. 2008. Diabetes data and trends [online]. Accessed February 23, 2008. URL: http://apps.nccd. cdc.gov/DDTSTRS/default.aspx.

Charbonnel B, Karasik A, Liu J, et al. 2006. Efficacy and safety of the dipeptidyl peptidase-4 inhibitor sitagliptin added to ongoing metformin therapy in patients with type 2 diabetes inadequately controlled with metformin alone. Diabetes Care, 29:2638-43.

Charpentier G. 2002. Oral combination therapy for type 2 diabetes. Diabetes Metab Res Rev, 18:S70-S76.

DeFronzo RA. 1999. Pharmacologic therapy for type 2 diabetes mellitus. Ann Intern Med, 131:281-303.

Del Prato S, Marchetti P. 2004. Beta- and alpha-cell dysfunction in type 2 diabetes. Horm Metab Res, 36:775-81.

Drucker DJ. 2003. Enhancing incretin action for the treatment of type 2 diabetes. Diabetes Care, 26:2929-40.

Drucker DJ, Nauck MA. 2006. The incretin system: glucagon-like peptide-1 receptor agonists and dipeptidyl peptidase- 4 inhibitors in type 2 diabetes. Lancet, 368:1696-706.

Gerich JE. 2002. Redefining the clinical management of type 2 diabetes: matching therapy to pathophysiology. Eur J Clin Invest, 32(S3):46-53.

Goldstein BJ, Feinglos MN, Lunceford JK, et al. 2007. Effect of initial combination therapy with sitagliptin, a dipeptidyl peptidase-4 inhibitor, and metformin on glycemic control in patients with type 2 diabetes. Diabetes Care, 30:1979-87.

Goodarzi MO, Bryer-Ash M. 2005. Metformin revisited: re-evaluation of its properties and role in the pharmacopoeia of modern antidiabetic agents. Diabetes Obes Metab, 7:654-65.

Herman GA, Stevens C, Van Dyck K, et al. 2005. Pharmacokinetics and pharmacodynamics of sitagliptin, an inhibitor of dipeptidyl peptidase IV, in healthy subjects: results from two randomized, double-blind, placebo-controlled studies with single oral doses. Clin Pharmacol Ther, 78:675-88.

Herman GA, Stein PP, Thornberry NA, et al. 2007. Dipeptidyl peptidase-4 inhibitors for the treatment of type 2 diabetes: focus on sitagliptin. Clin Pharmacol Ther, 81:761-7.

Hermansen K, Kipnes M, Luo E, et al. 2007. Efficacy and safety of the dipeptidyl peptidase- 4 inhibitor, sitagliptin, in patients wtih type 2 diabetes mellitus inadequately controlled on glimepiride alone or on glimepiride and metformin. Diabetes Obes Metab, 9:733-45.

Holst JJ. 2002. Therapy of type 2 diabetes mellitus based on the actions of glucagon-like peptide-1. Diabetes Metab Res Rev, 18:430-41.

[IDF] International Diabetes Federation. 2008. Global guideline for type 2 diabetes [online]. Accessed February 23, 2008. URL: http://www.idf. org/home/index.cfm?node=1457. 
Inzucchi SE. 2002. Oral antihyperglycemic therapy for type 2 diabetes. JAMA, 287:360-72.

Kahn SE. 2003. The relative contributions of insulin resistance and beta-cell dysfunction to the pathophysiology of type 2 diabetes. Diabetologia, 46:3-19.

Kahn SE, Haffner SM, Heise MA, et al. 2006. Glycemic durability of rosiglitazone, metformin, or glyburide monotherapy. NEJM, 355:2427-43.

Knowler WC, Barrett-Connor E, Fowler SE, et al. 2002. Reduction in the incidence of type 2 diabetes with lifestyle intervention or metformin. N Engl J Med, 346:393-403.

Lenhard JM, Croom DK, Minnick DT. 2004. Reduced serum dipeptidyl peptidase-IV after metformin and pioglitazone treatments. Biochem Biophys Res Commun, 324:92-7.

Mannucci E, Ognibene A, Cremasco F, et al. 2001. Effect of metformin on glucagon-like peptide 1 (GLP-1) and leptin levels in obese nondiabetic subjects. Diabetes Care, 24:489-94.

Merck and Co, Inc. 2007. Januvia prescribing information [online]. Accessed February 23, 2008. URL: http://www.januvia.com.

Mu J, Woods J, Zhou Y, et al. 2006. Chronic inhibition of dipeptidyl peptidase-4 with a sitagliptin analog preserves pancreatic beta-cell mass and function in a rodent model of type 2 diabetes. Diabetes, 55:1695-704.

Nathan DM, Buse JB, Davidson MB, et al. 2006. Management of hyperglycemia in type 2 diabetes: a consensus algorithm for the initiation and adjustment of therapy. Diabetes Care, 29:1963-72.

Nathan DM, Holman RR, Buse JB, et al. 2008. Management of hyperglycemia in type 2 diabetes: a consensus algorithm for the initiation and adjustment of therapy. Diabetes Care, 31:173-5.

Nauck M, Stockmann F, Ebert R, et al. 1986. Reduced incretin effect in type 2 (non-insulin-dependent) diabetes. Diabetologia, 29:46-52.

Nauck MA, Kleine N, Orskov C, et al. 1993. Normalization of fasting hyperglycemia by exogenous glucagon-like peptide 1 (7-36 amide) in type 2 (non-insulin-dependent) diabetic patients. Diabetologia, 36:741-4.

Nauck MA, Meininger G, Sheng D, et al. 2007. Efficacy and safety of the dipeptidyl peptidase-4 inhibitor, sitagliptin, compared with the sulfonylurea, glipizide, in patients with type 2 diabetes inadequately controlled on metformin alone: a randomized, double-blind, non-inferiority trial. Diabetes Obes Metab, 9:194-205.

Nissen SE, Wolski K. 2007. Effect of rosiglitazone on the risk of myocardial infarction and death from cardiovascular causes. $N$ Engl J Med, 356:2457-71.
Raz I, Hanefeld M, Xu L, et al. 2006. Efficacy and safety of the dipeptidyl peptidase-4 inhibitor sitagliptin as monotherapy in patients with type 2 diabetes mellitus. Diabetologia, 49:2564-571.

Reitman ML, Schadt EE. 2007. Pharmacogenetics of metformin response: a step in the path toward personalized medicine. J Clin Invest, $117: 1226-9$

Riddle MC. 2005. Glycemic management of type 2 diabetes: an emerging strategy with oral agents, insulins, and combinations. Endocrinol Metab Clin North Am, 34:77-98.

Rosenstock J, Brazg R, Andryuk PJ, et al. 2006. Efficacy and safety of the dipeptidyl peptidase-4 inhibitor sitagliptin added to ongoing pioglitazone therapy in patients with type 2 diabetes: a 24 -week, multicenter, randomized, double-blind, placebo-controlled, parallel-group study. Clin Therapeutics, 28:1556-68.

Salpeter S, Greyber E, Pasternak G, et al. 2006. Risk of fatal and nonfatal lactic acidosis with metformin use in type 2 diabetes mellitus. Cochrane Database Syst Rev, 1:CD002967.

Saydah SH, Fradkin J, Cowie CC. 2004. Poor control of risk factors for vascular disease among adults with previously diagnosed diabetes. JAMA, 291:335-42.

Scheen AJ. 1996. Clinical pharmacokinetics of metformin. Clin Pharmacokinetics, 30:359-71.

Stratton IM, Adler AI, Neil HA, et al. 2000. Association of glycemia with macrovascular and microvascular complications of type 2 diabetes (UKPDS 35): prospective observational study. BMJ, 321:405-12.

Turner RC, Cull CA, Frighi V, et al. 1999. Glycemic control with diet, sulfonylureas, metformin, or insulin in patients with type 2 diabetes mellitus: progressive requirement for multiple therapies (UKPDS 49). JAMA, 281:2005-12.

[UKPDS] UK Prospective Diabetes Study Group. 1995. U.K. prospective diabetes study 16: overview of 6 years' therapy of type II diabetes: a progressive disease. Diabetes, 44:1249-58.

[UKPDS] UK Prospective Diabetes Study (UKPDS) Group. 1998. Effect of intensive blood-glucose control with metformin on complications in overweight patients with type 2 diabetes (UKPDS 34). Lancet, 352:854-65.

[WHO] World Health Organization. 2008. Diabetes facts [online]. Accessed February 23, 2008. URL: http://www.who.int/mediacentre/factsheets/ fs312/en/index.html. 
